\title{
Active Damping Control using Optimal Integral Force Feedback
}

\author{
Yik R. Teo, Andrew J. Fleming
}

\begin{abstract}
This article shows an improvement to Integral Force Feedback (IFF) for active damping control of precision mechanisms. The benefits of IFF include robustness, guaranteed stability and simplicity. However, the damping performance depends on the stiffness of the system; hence, some systems cannot be adequately controlled. In this article, an extension to the classical integral force feedback control scheme is proposed. The new method achieves arbitrary damping for any mechanical system by introducing a feed-through term in the system.
\end{abstract}

\section{INTRODUCTION}

The speed and resolution of many scientific and industrial machines are limited by the presence of lightly damped mechanical resonances. Examples include scanning probe microscopy [1]-[5], nanofabrication [6], precision optics [7] and aerospace systems [8].

Traditional passive damping methods have includes viscoelastic damping and tuned-mass absorbers; however, these methods can be bulky and may not perform well at low frequencies. In contrast, active damping is an alternative method to increase the performance. Active damping control utilizes a sensor and feedback loop to artificially increase the damping ratio of a system. A number of successful damping control techniques include Positive Position Feedback (PPF) [9], polynomial based control [10], shunt control [11]-[13], resonant control [14], Force Feedback [15]-[18], and Integral Resonance Control (IRC) [19][21]. Among these techniques, PPF controllers, velocity feedback controllers, force feedback controllers, and IRC controllers have been shown to guarantee stability when the plant is strictly negative imaginary [22]. Optimal controllers with automatic synthesis have also been successfully applied to damping control applications, for example, robust $\mathscr{H}_{\infty}$ controllers [23], [24].

In references [15]-[18] integral force feedback (IFF) is described for vibration control and positioning applications. This technique utilizes a force sensor and integral controller to directly augment the damping of a mechanical system. The major advantages of IFF are the simplicity of the controller, guaranteed stability and excellent performance robustness. However, the maximum damping achievable with IFF is a function of the system properties, in particular the system stiffness relative to the actuator stiffness. Hence, some systems can be critically damped using IFF while other exhibit negligible damping improvement.

Yik R. Teo and Andrew J. Fleming are with Precision Mechatronics $\mathrm{Lab}$ at the School of Electrical Engineering and Computer Science, The University of Newcastle, 2308, Callaghan, NSW, Australia. (yik.teo, andrew.fleming) @newcastle.edu.au

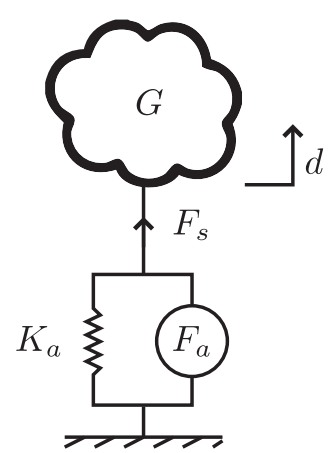

Fig. 1. Structure $G$ with a piezoelectric transducer

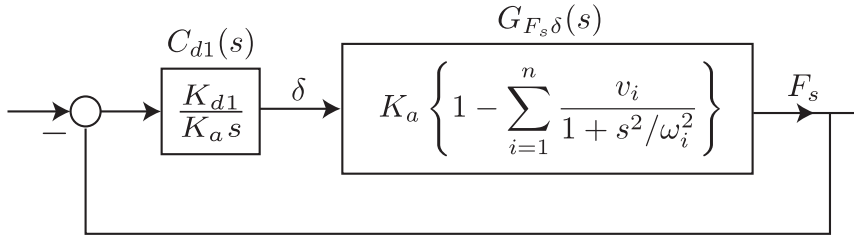

Fig. 2. Block Diagram of the Classical Integral Force Feedback (CIFF)

In this work, an extension to IFF is described for an arbitrary damping ratio to be achieved for any mechanical system. The modification amounts to replacing the integral controller with a first-order equivalent low-pass filter. Although the additional complexity is negligible, the damping performance is significantly improved. This is an exceptional result that allows integral force feedback to be extended to systems that were not previously suited.

\section{Classical Integral Force Feedback (CIFF)}

Integral Force Feedback (IFF) control has been widely applied for augmenting the damping of flexible structures [16]. The feedback law is simple to implement and under common circumstances provides excellent damping performance with guaranteed stability. Fig. 1 illustrates a structure $G$ equipped with a piezoelectric actuator that produces a force $F_{a}$ with internal stiffness $K_{a}$. A force sensor is collocated with the piezoelectric actuator and measures the axial force $F_{s}$ acting on the system $G$. The variable $d$ represents the mechanical displacement.

The classical integral force feedback controller (CIFF) has a block diagram representation illustrated in Fig. 2. The transfer function between the unconstrained piezo expansion $\delta$ to the sensor force $F_{s}$ is [25]

$$
G_{F_{s} \delta}(s)=\frac{F_{s}}{\delta}=K_{a}\left\{1-\sum_{i=1}^{n} \frac{v_{i}}{1+s^{2} / \omega_{i}^{2}}\right\},
$$




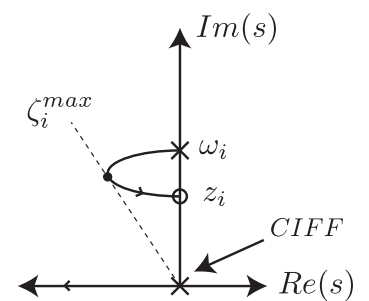

(a) Classical method.

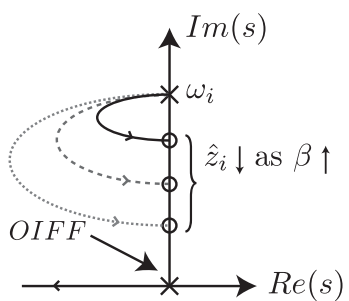

(b) Optimal method.
Fig. 3. Typical root locus plots.

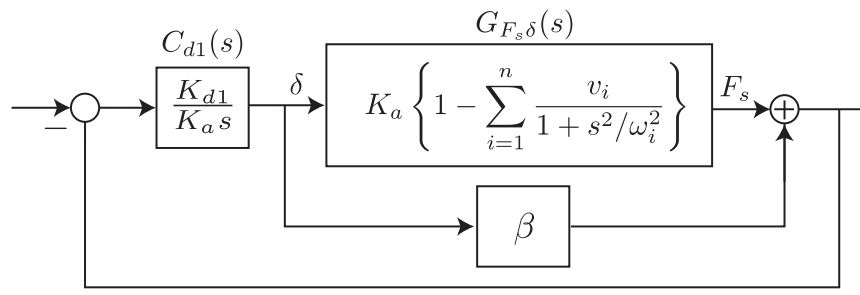

(a) Block Diagram of the OIFF with new feed-through term $\beta$.

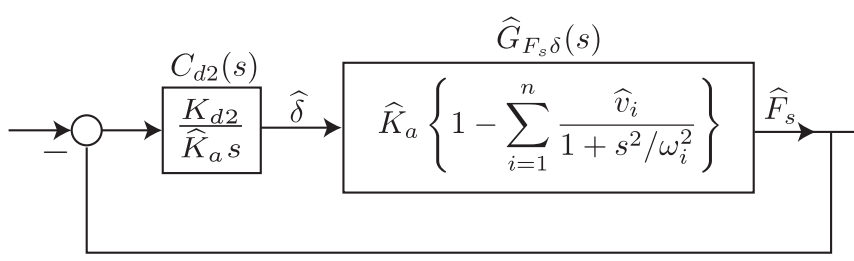

(b) Block Diagram of the OIFF in the classical form with $\widehat{K}_{a}$ and $\widehat{v}_{i}$.

Fig. 4. Block Diagrams of Optimal Integral Force Feedback (OIFF).

where $\omega_{i}$ is the natural frequency of the system and $v_{i}$ is the fraction of modal strain energy for the $i^{t h}$ mode. The modal zeros are [16],

$$
z_{i}^{2}=\omega_{i}^{2}\left(1-v_{i}\right)
$$

The integral force feedback controller is

$$
C_{d 1}(s)=\frac{K_{d 1}}{K_{a} s}
$$

where $K_{d}$ is the gain of controller. The maximum modal damping is

$$
\zeta_{i}^{\max }=\frac{\omega_{i}-z_{i}}{2 \omega_{i}}
$$

and is achieved for

$$
K_{d 1}=\omega_{i} \sqrt{\frac{\omega_{i}}{z_{i}}} .
$$

The root locus plot corresponding to CIFF is shown in Fig. 3(a). A key limitation of the classical method is that the maximum modal damping (4) depends on the distance between the system poles $\omega_{i}$ and modal zeros $z_{i}$. If the distance between the pole and zero is small, the maximum damping achievable with CIFF is not significant.

\section{Optimal Integral Force FeEdback (OIFF)}

In this work, we extend the classical integral force feedback methodology by introducing a feed-through term $\beta$ in the system as shown in Fig. 4(a). The new system can be converted into the classical form by equating the systems in Fig. 4(a) and Fig. 4(b), that is

$$
\begin{gathered}
K_{a}\left\{1-\sum_{i=1}^{n} \frac{v_{i}}{1+s^{2} / \omega_{i}^{2}}\right\}+\beta=\widehat{K}_{a}\left\{1-\sum_{i=1}^{n} \frac{\widehat{v}_{i}}{1+s^{2} / \omega_{i}^{2}}\right\}, \\
K_{a}+\beta-\sum_{i=1}^{n} \frac{K_{a} v_{i}}{1+s^{2} / \omega_{i}^{2}}=\widehat{K}_{a}-\sum_{i=1}^{n} \frac{\widehat{K}_{a} \widehat{v}_{i}}{1+s^{2} / \omega_{i}^{2}} .
\end{gathered}
$$

From (6) we obtain the new expressions

$$
\begin{aligned}
\widehat{K}_{a} & =K_{a}+\beta, \\
\widehat{v}_{i} & =\frac{K_{a} v_{i}}{\widehat{K}_{a}}, \\
& =\frac{K_{a} v_{i}}{K_{a}+\beta} .
\end{aligned}
$$

The transfer function from $\widehat{\delta}$ to $\widehat{F}_{S}$ is

$$
\widehat{G}_{F_{s} \delta}(s)=\widehat{K}_{a}\left\{1-\sum_{i=1}^{n} \frac{\widehat{v}_{i}}{1+s^{2} / \omega_{i}^{2}}\right\} .
$$

The modal zeros are now a function of $\beta$

$$
\widehat{z}_{i}(\beta)=\sqrt{\omega_{i}^{2}\left(1-\frac{K_{a}}{K_{a}+\beta} v_{i}\right)} .
$$

This results in an extra degree of freedom that allows the position of the zeros to be modified. As $\beta$ decreases, the zeros move closer to the real axis, under the condition that $K_{a}\left(v_{i}-1\right)<\beta<0$ is satisfied. The integral force feedback controller is

$$
C_{d 2}(s)=\frac{K_{d 2}}{\widehat{K}_{a} s},
$$

The root locus plot for a typical OIFF system is shown in Fig. 3(b). Notice that the location of the zero changes with respect to $\beta$. The new maximum modal damping is

$$
\widehat{\zeta}_{i}^{\max }=\frac{\omega_{i}-\widehat{z}_{i}(\beta)}{2 \widehat{z}_{i}(\beta)},
$$

which corresponds to the new optimal gain

$$
K_{d 2}=\omega_{i} \sqrt{\frac{\omega_{i}}{\widehat{z}_{i}(\beta)}} .
$$

On the other hand, given a desired modal damping $\zeta_{d}$, the value of $\beta$ required is

$$
\beta=-K_{a}+\frac{K_{a} v_{i}\left(2 \zeta_{d}+1\right)^{2}}{4 \zeta_{d}\left(1+\zeta_{d}\right)} .
$$

\section{CAse Study}

In this section, the performance of CIFF and OIFF is evaluated on a simple mechanical system. 


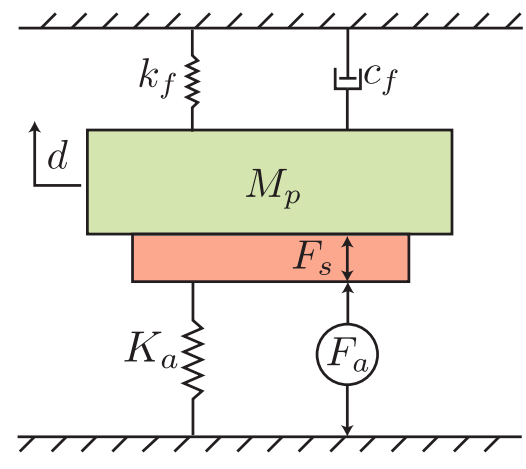

Fig. 5. Mechanical diagram of a second-order mechanical system where $F_{s}$ is the measured force acting between the actuator and the mass in the vertical direction.

\section{A. Mechanical Dynamics and System Properties}

Fig. 5 shows a second-order mechanical system with mass $M_{p}=250 \mathrm{~g}$, flexure stiffness $k_{f}=300 \mathrm{~N} / \mu \mathrm{m}$, actuator stiffness $K_{a}=100 \mathrm{~N} / \mu \mathrm{m}$ and flexure damping $c_{f}=10 \mathrm{~N} / \mathrm{ms}^{-1}$.

The equation of motion for this system is

$$
M_{p} \ddot{d}+c_{f} \dot{d}+\left(K_{a}+k_{f}\right) d=F_{a},
$$

where $M_{p}$ is the mass of the platform and the stiffness and damping coefficient of the flexures are denoted by $k_{f}$ and $c_{f}$ respectively. The force of the actuator is $F_{a}$ and the stiffness is $K_{a}$. A force sensor is collocated with the actuator and measures the load force $F_{s}$.

The configuration of the system is such that the actuator and flexure appear mechanically in parallel, hence, the stiffness coefficients can be grouped together $k=K_{a}+k_{f}$. This simplifies the equation of motion (15) to

$$
M_{p} \ddot{d}+c_{f} \dot{d}+k=F_{a} .
$$

The transfer function from actuator force $F_{a}$ to the displacement of the mass $d$ is

$$
\begin{aligned}
G_{d F_{a}}(s) & =\frac{d}{F_{a}}, \\
& =\frac{1}{M_{p} s^{2}+c_{f} s+k} .
\end{aligned}
$$

The sensor force $F_{S}$ can be written as

$$
\begin{aligned}
F_{s} & =F_{a}-d K_{a}, \\
& =F_{a}-K_{a} F_{a} G_{d F_{a}}(s), \\
& =F_{a}\left(1-K_{a} G_{d F_{a}}(s)\right) .
\end{aligned}
$$

The transfer function between the applied force $F_{a}$ and measured force $F_{S}$ is found by rearranging (18).

$$
\begin{aligned}
G_{F_{s} F_{a}}(s) & =\frac{F_{s}}{F_{a}}, \\
& =1-K_{a} G_{d F_{a}}(s) .
\end{aligned}
$$

The force developed by the actuator $F_{a}$ is

$$
F_{a}=K_{a} \delta
$$

recall that $\delta$ is the unconstrained piezo expansion. Substituting (20) into (19), we obtain the transfer function from the

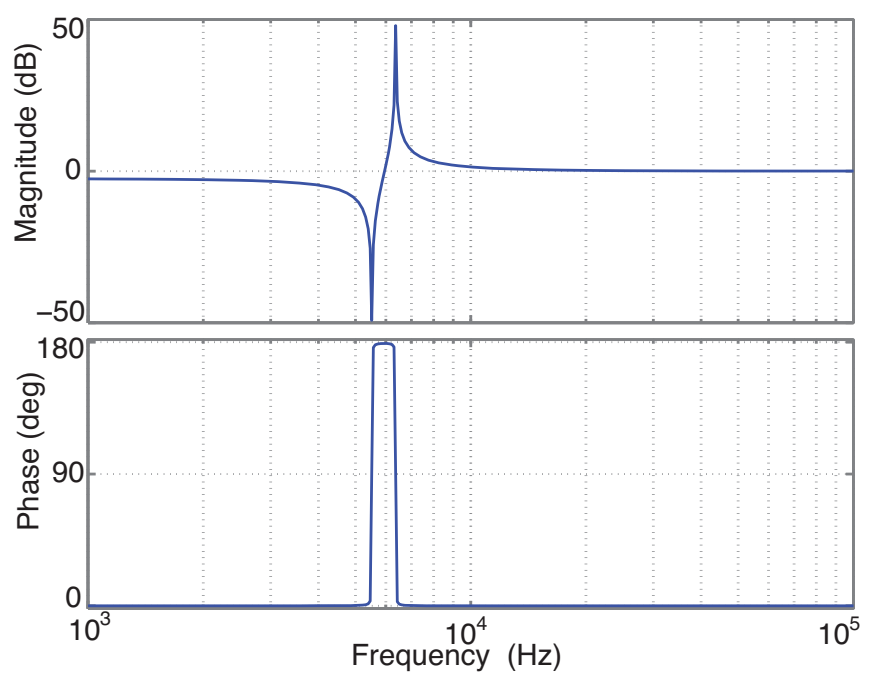

Fig. 6. Case Study: Frequency Response of $G_{F_{s} F_{a}}(s)$.

unconstrained piezo expansion $\delta$ to the force of the sensor $F_{S}$

$$
\begin{aligned}
G_{F_{s} \delta} & =\frac{F_{s}}{\delta}, \\
& =K_{a} \frac{F_{s}}{F_{a}}, \\
& =K_{a}\left(1-K_{a} G_{d F_{a}}(s)\right) .
\end{aligned}
$$

A valid assumption is that the effect of the damping in the flexure $c_{f}$ is small and thus negligible.

The imaginary parts of the open-loop poles and zeros are

$$
\begin{aligned}
\omega_{1} & =\sqrt{\frac{k}{M_{p}}}, \\
& =\sqrt{\frac{K_{a}+k_{f}}{M_{p}}}, \\
z_{1} & =\sqrt{\frac{k_{f}}{M_{p}}} .
\end{aligned}
$$

For this system the open-loop poles and zeros of the system are $\omega_{1}=6.37 \mathrm{kHz}$ and $z_{1}=5.5 \mathrm{kHz}$.

\section{B. Damping Control Design}

1) Classical Integral Force Feedback (CIFF): The openloop frequency response of $G_{F_{s} F_{a}}(s)$ is shown in Fig. 6. One key observation is that its phase response of the system lies between 0 and $180^{\circ}$. This is a general property of flexible structures with inputs and outputs proportional to the applied and measured forces. Recall that the classical integral force feedback controller is

$$
C_{d 1}(s)=\frac{K_{d 1}}{K_{a} s} .
$$

The integral controller has a constant phase lag of $90^{\circ}$ so the loop-gain of the system lies between -90 and $90^{\circ}$. Hence, the closed-loop system has an infinite gain margin and phase margin of $90^{\circ}$. The solution for the optimal feedback gain 


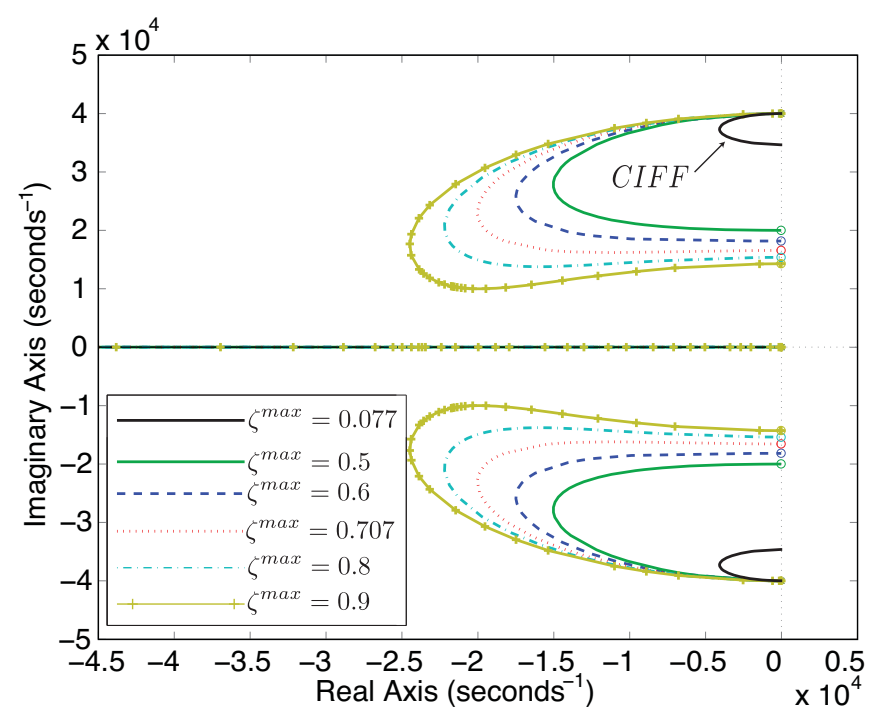

Fig. 7. Case Study: Root locus of the system using CIFF and OIFF at different values of $\zeta^{\max }$.

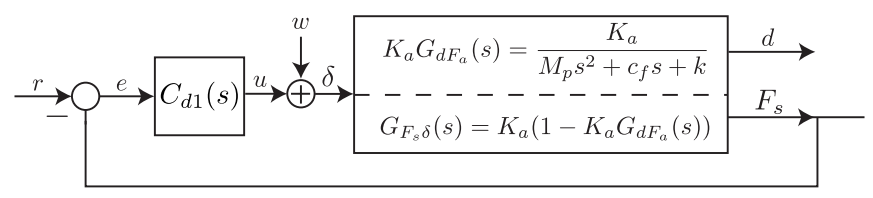

Fig. 8. Detail Block Diagram of the CIFF system for analysis

$K_{d}$ has already been derived in [16] and further generalised for nanopositioning systems in [17]. The optimal feedback gain $K_{d}$ and corresponding maximum closed-loop damping ratio $\zeta_{1}^{\max }$ are

$$
\begin{aligned}
K_{d 1} & =\omega_{1} \sqrt{\frac{\omega_{1}}{z_{1}}}, \\
& =4.3 \times 10^{4},
\end{aligned}
$$

and

$$
\begin{aligned}
\zeta_{1}^{\max } & =\frac{\omega_{1}-z_{1}}{2 z_{1}}, \\
& =0.077 .
\end{aligned}
$$

The numerical root-locus plot in Fig. 7 validate these values. The numerically optimal gain is $4.57 \times 10^{4}$ and the corresponding damping ratio is 0.077 . This correlates closely with the predicted values which supports the accuracy of the assumptions made in deriving the optimal gain.

Fig. 8 shows a detailed block diagram of the system with sensor force $F_{S}$ and platform displacement $d$ as outputs. A disturbance $w$ is also considered. The transfer function from the disturbance $w$ to the sensor force $F_{S}$ is

$$
\begin{aligned}
G_{F_{s} w}(s) & =\frac{F_{s}}{w}, \\
& =\frac{G_{F_{s}} \delta}{1+C_{d} G_{F_{s}} \delta} .
\end{aligned}
$$

The simulated open-loop and closed-loop frequency responses of (26) are plotted in Fig. 9.

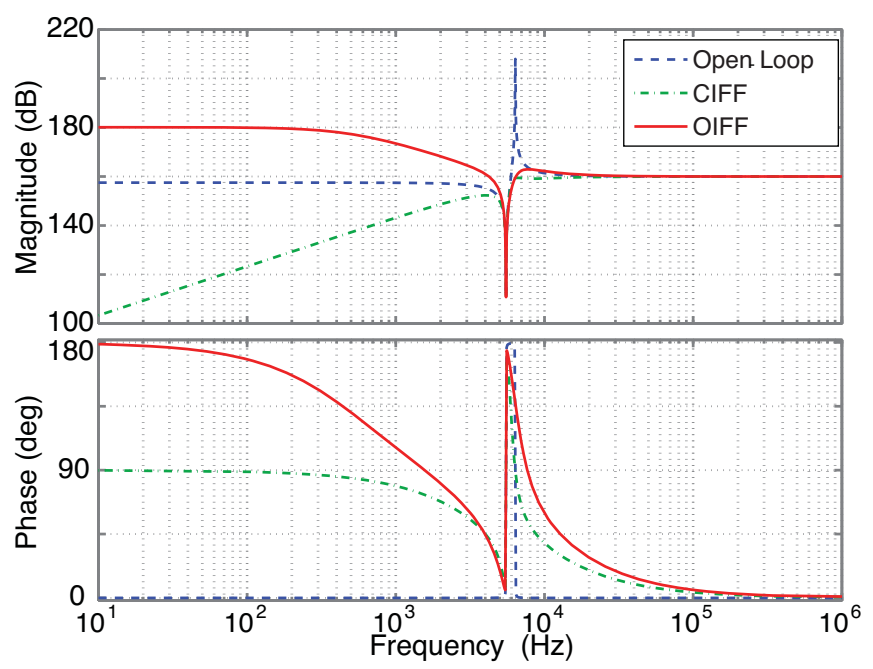

Fig. 9. Case Study: Frequency response from the input disturbance $w$ to the sensor force $F_{s}$.

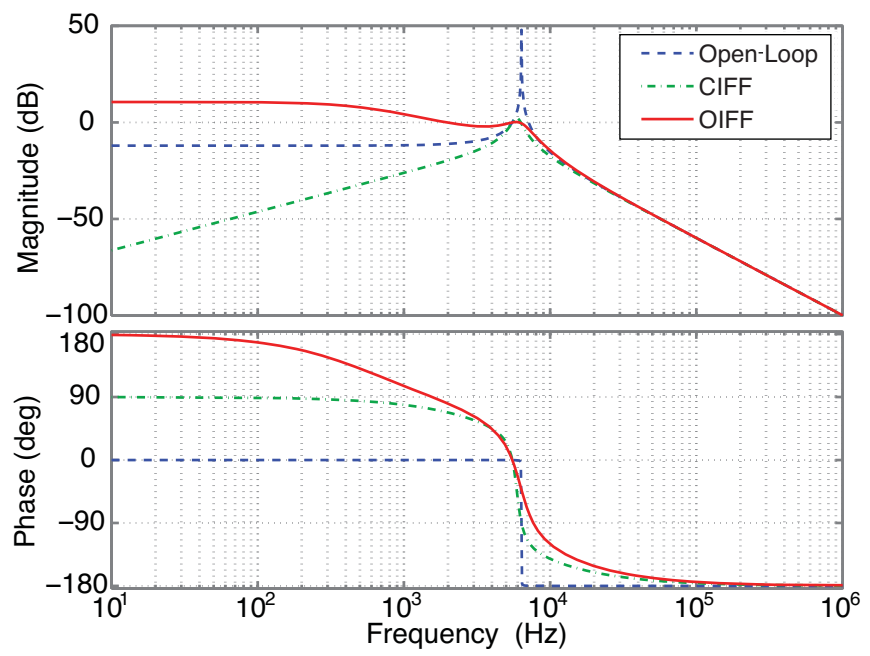

Fig. 10. Case Study: Frequency response from the input disturbance $w$ to the displacement of the platform $d$.

The transfer function from the disturbance $w$ to the displacement of the platform $d$ is

$$
\begin{aligned}
G_{d w}(s) & =\frac{d}{w}, \\
& =\frac{K_{a} G_{d F_{a}}}{1+C_{d} G_{F_{s}} \delta} .
\end{aligned}
$$

The simulated open-loop and closed-loop frequency responses of (27) are plotted in Fig. 10.

2) Optimal Integral Force Feedback (OIFF): The CIFF method can be extended to include the feed-through term $\beta$ as illustrated in Fig. 4(a). The equivalent controller $\widehat{C}_{d}(s)$ is found by equating the systems in Fig. 4(b) and Fig. 11,

$$
\widehat{C}_{d}(s)=\frac{C_{d 2}(s)}{1+C_{d 2}(s) \beta} \text {. }
$$

For the case study, the relationship between $\beta$ and $\zeta$ described in (14) is plotted in Fig. 12. The maximum 


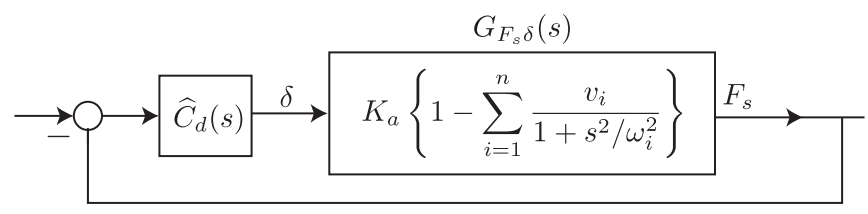

Fig. 11. Block Diagram of the example system using OIFF.

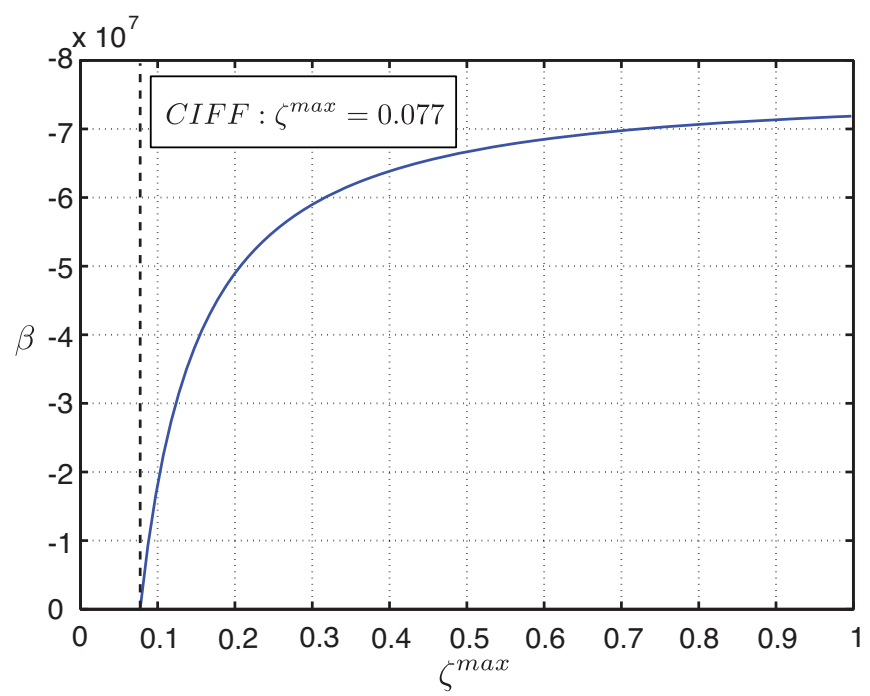

Fig. 12. Case Study: The relationship between $\beta$ and $\zeta^{\max }$ for OIFF.

modal damping with CIFF is 0.077; however, with OIFF, the maximum modal damping can be varied from 0.077 to 1 at different values of $\beta$.

The root locus of the system is shown in Fig. 7. The optimal feedback gain, maximum damping ratio and corresponding value of $\beta$ is given in Table I. These values can be validated by the numerical root-locus plot in Fig. 7 and is summarize in Table I. These values correlates closely with the predicted values which supports the accuracy of the assumptions made in deriving the optimal gain.

Fig. 13 shows a detailed block diagram of the system with sensor force $F_{S}$ and platform displacement $d$ as outputs. A disturbance $w$ is also considered. The transfer function from the disturbances $w$ to the sensor force $F_{s}$ is

$$
\begin{aligned}
G_{F_{s} w}(s) & =\frac{F_{s}}{w}, \\
& =\frac{G_{F_{s}} \delta}{1+\widehat{C}_{d} G_{F_{s}} \delta} .
\end{aligned}
$$

The simulated open-loop and closed-loop frequency responses of (29) is shown in Fig. 9.

The closed-loop transfer function measured from the reference, $r$, to the sensor force, $F_{S}$, is

$$
\begin{aligned}
G_{F_{s} r}(s) & =\frac{F_{s}}{r}, \\
& =\frac{\widehat{C}_{d} G_{F_{s}} \delta}{1+\widehat{C}_{d} G_{F_{s}} \delta} .
\end{aligned}
$$

TABLE I

COMPARISON BETWEEN ANALYTIC AND NUMERICALLY OBTAINED DAMPING RATIO $\zeta^{\max }$ AND FEEDBACK GAIN $K_{d 2}$.

\begin{tabular}{|c|c|c|c|c|}
\cline { 2 - 5 } \multicolumn{1}{c|}{} & \multicolumn{2}{c|}{ Analytic } & \multicolumn{2}{c|}{ Numerical } \\
\hline$\beta$ & $\zeta^{\max }$ & $K_{d 2}$ & $\zeta^{\max }$ & $K_{d 2}$ \\
\hline$-6.67 \times 10^{7}$ & 0.500 & $5.65 \times 10^{4}$ & 0.501 & $5.57 \times 10^{4}$ \\
\hline$-6.85 \times 10^{7}$ & 0.600 & $5.93 \times 10^{4}$ & 0.601 & $5.94 \times 10^{4}$ \\
\hline$-6.98 \times 10^{7}$ & 0.707 & $6.21 \times 10^{4}$ & 0.708 & $6.23 \times 10^{4}$ \\
\hline$-7.07 \times 10^{7}$ & 0.800 & $6.45 \times 10^{4}$ & 0.801 & $6.49 \times 10^{4}$ \\
\hline$-7.13 \times 10^{7}$ & 0.900 & $6.69 \times 10^{4}$ & 0.902 & $6.70 \times 10^{4}$ \\
\hline
\end{tabular}

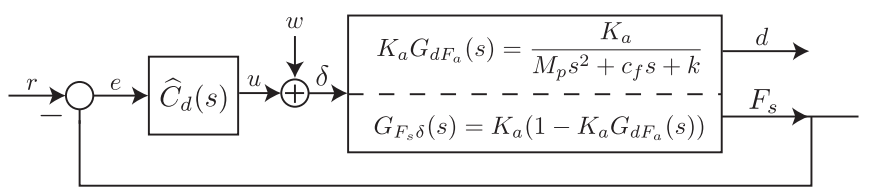

Fig. 13. Detail Block Diagram of the OIFF system for analysis

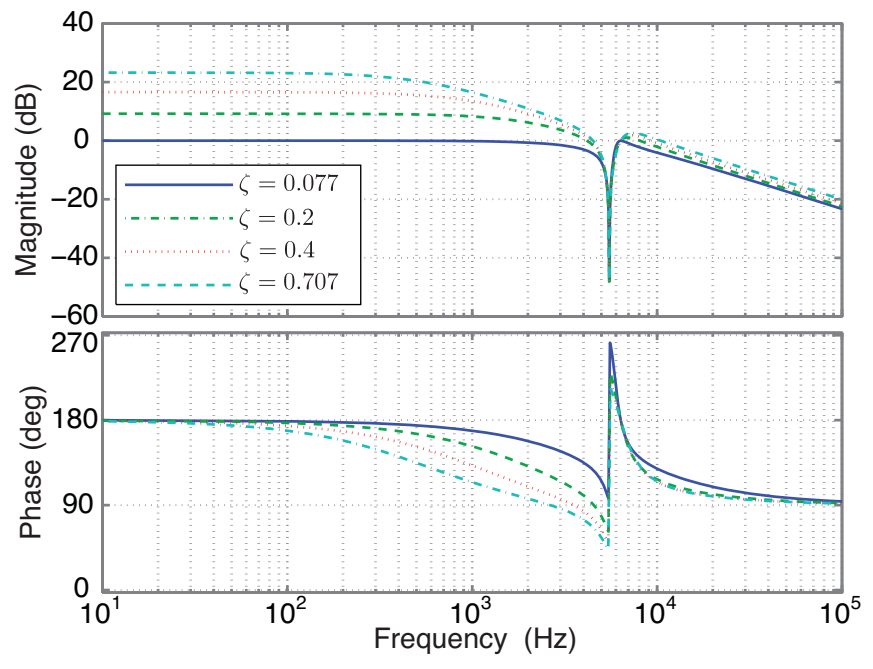

Fig. 14. Case Study: Frequency response from the input disturbance $w$ to the control action $u$ as $\beta$ is decreased.

when $s=0$

$$
\begin{aligned}
& G_{F_{s} r}(0)=\frac{\widehat{C}_{d} G_{F_{s} \delta}(0)}{1+\widehat{C}_{d} G_{F_{s} \delta}(0)}, \\
& =\frac{G_{F_{s} \delta}(0)}{G_{F_{s}}(0)+\beta} .
\end{aligned}
$$

This shows that the DC gain of the closed-loop increases as $\beta$ decreases such that $K_{a}\left(v_{i}-1\right)<\beta<0$ holds. Recall that the maximum damping ratio of the closed-loop system increases as $\beta$ is decrease.

The transfer function from the disturbance $w$ to the displacement of the platform $d$ is

$$
\begin{aligned}
G_{d w}(s) & =\frac{d}{w}, \\
& =\frac{K_{a} G_{d F_{a}}}{1+\widehat{C}_{d} G_{F_{s}} \delta} .
\end{aligned}
$$

The simulated open-loop and closed-loop frequency responses of (32) are plotted in Fig. 14. The frequency response 
of this transfer function is shown in Fig. 14. The sensitivity of the control action toward input disturbance increases as the desired damping ratio is increased.

\section{CONClusion}

This article describes an extension to integral force feedback control that allows arbitrary mechanical damping to be achieved for any mechanical system. An additional feedthrough term is added to the system to provide an extra degree of freedom that can be used to arbitrarily manipulate the modal zeros and maximum damping.

Simulation results on a simple mechanical system demonstrate an increase in the maximum achievable damping from 0.077 to 1 using integral force feedback. This result will allow high-performance mechanical systems to be critically damped with a first-order control law.

Future work will include experimental application, extension to systems with multiple actuators, and modeling using a negative imaginary framework.

\section{REFERENCES}

[1] N. Jalili and K. Laxminarayana, "A review of atomic force microscopy imaging systems: application to molecular metrology and biological sciences," Mechatronics, vol. 14, no. 8, pp. 907-945, October 2004.

[2] A. J. Fleming, B. J. Kenton, and K. K. Leang, "Bridging the gap between conventional and video-speed scanning probe microscopes," Ultramicroscopy, vol. 110, no. 9, pp. 1205-1214, August 2010.

[3] K. K. Leang and A. J. Fleming, "High-speed serial-kinematic AFM scanner: design and drive considerations," Asian Journal of Control, vol. 11, no. 2, pp. 144-153, March 2009.

[4] A. J. Fleming and Y. K. Yong, "Piezoelectric actuators with integrated high voltage power electronics," IEEE/ASME Transactions on Mechatronics, vol. (In Press), 2014.

[5] A. J. Fleming and K. K. Leang, Design, Modeling and Control of Nanopositioning Systems. London, UK: Springer, 2014.

[6] A. Ferreira and C. Mavroidis, "Virtual reality and haptics for nanorobotics," IEEE Robotics and Automation Magazine, vol. 13, no. 3, pp. 78-92, September 2006.

[7] L. Vaillon and C. Philippe, "Passive and active microvibration control for very high pointing accuracy space systems," Smart Materials and Structures, vol. 8, no. 6, December 1999.

[8] V. Giurgiutiu, "Review of smart-materials actuation solutions for aeroelastic and vibration control," Journal of Intelligent Material Systems and Structures, vol. 11, pp. 525-544, July 2000.

[9] J. L. Fanson and T. K. Caughey, "Positive position feedback control for large space structures," AIAA Journal, vol. 28, no. 4, pp. 717-724, 1990.

[10] S. S. Aphale, B. Bhikkaji, and S. O. R. Moheimani, "Minimizing scanning errors in piezoelectric stack-actuated nanopositioning platforms," IEEE Transactions on Nanotechnology, vol. 7, no. 1, pp. 7990, January 2008.

[11] A. J. Fleming and S. O. R. Moheimani, "Sensorless vibration suppression and scan compensation for piezoelectric tube nanopositioners," IEEE Transactions on Control Systems Technology, vol. 14, no. 1, pp. 33-44, January 2006.

[12] A. J. Fleming, S. Behrens, and S. O. R. Moheimani, "Optimization and implementation of multi-mode piezoelectric shunt damping systems," IEEE/ASME Transactions on Mechatronics, vol. 7, no. 1, pp. 87-94, March 2002.

[13] A. A. Eielsen and A. J. Fleming, "Passive shunt damping of a piezoelectric stack nanopositioner," in Proc. American Control Conference, Baltimore, MD, June 2010, pp. 4963-4968.

[14] A. Sebastian, A. Pantazi, S. O. R. Moheimani, H. Pozidis, and E. Eleftheriou, "A self servo writing scheme for a MEMS storage device with sub-nanometer precision," in Proc. IFAC World Congress, Seoul, Korea, July 2008, pp. 9241-9247.

[15] A. Preumont, J. P. Dufour, and C. Malekian, "Active damping by a local force feedback with piezoelectric actuators." AIAA Journal of Guidance and Control, vol. 15, no. 2, 1992.
[16] A. Preumont, Mechatronics, Dynamics of electromechanical and piezoelectric systems. Dordrecht, The Netherlands: Springer, 2006.

[17] A. J. Fleming, "Nanopositioning system with force feedback for highperformance tracking and vibration control," IEEE Transactions on Mechatronics, vol. 15, no. 3, pp. 433-447, June 2010.

[18] A. J. Fleming and K. K. Leang, "Integrated strain and force feedback for high performance control of piezoelectric actuators," Sensors and Actuators A, vol. 161, no. 1-2, pp. 256-265, June 2010.

[19] S. S. Aphale, A. J. Fleming, and S. O. R. Moheimani, "Integral resonant control of collocated smart structures," IOP Smart materials and Structures, vol. 16, pp. 439-446, April 2007.

[20] B. Bhikkaji and S. O. R. Moheimani, "Integral resonant control of a piezoelectric tube actuator for fast nanoscale positioning," IEEE/ASME Transactions on Mechatronics, vol. 13, no. 5, pp. 530-537, October 2008.

[21] A. J. Fleming, S. S. Aphale, and S. O. R. Moheimani, "A new method for robust damping and tracking control of scanning probe microscope positioning stages," IEEE Transactions on Nanotechnology, vol. 9, no. 4, pp. 438-448, September 2010.

[22] I. R. Petersen and A. Lanzon, "Feedback control of negative-imaginary systems," Control Systems, IEEE, vol. 30, no. 5, pp. 54 -72, oct. 2010.

[23] S. Salapaka, A. Sebastian, J. P. Cleveland, and M. V. Salapaka, "High bandwidth nano-positioner: A robust control approach," Review of Scientific Instruments, vol. 75, no. 9, pp. 3232-3241, September 2002.

[24] A. Sebastian and S. M. Salapaka, "Design methodologies for robust nano-positioning," IEEE Transactions on Control Systems Technology, vol. 13, no. 6, pp. 868-876, November 2005.

[25] A. Preumont, B. de Marneffe, A. Deraemaeker, and F. Bossens, "The damping of a truss structure with a piezoelectric transducer," Computers and Structures, vol. 86, 2007. 